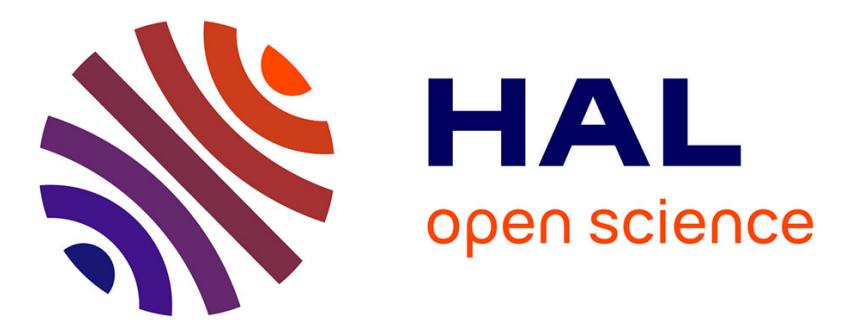

\title{
Filamentary nanosecond surface dielectric barrier discharge. Plasma properties in the filaments
}

S.A. Shcherbanev, Chenyang Ding, Svetlana Starikovskaia, N.A. Popov

\section{To cite this version:}

S.A. Shcherbanev, Chenyang Ding, Svetlana Starikovskaia, N.A. Popov. Filamentary nanosecond surface dielectric barrier discharge. Plasma properties in the filaments. Plasma Sources Science and Technology, 2019, 28 (6), pp.065013. 10.1088/1361-6595/ab2230 . hal-02272114

\section{HAL Id: hal-02272114 \\ https://hal.science/hal-02272114}

Submitted on 3 Sep 2020

HAL is a multi-disciplinary open access archive for the deposit and dissemination of scientific research documents, whether they are published or not. The documents may come from teaching and research institutions in France or abroad, or from public or private research centers.
L'archive ouverte pluridisciplinaire HAL, est destinée au dépôt et à la diffusion de documents scientifiques de niveau recherche, publiés ou non, émanant des établissements d'enseignement et de recherche français ou étrangers, des laboratoires publics ou privés. 


\title{
Filamentary nanosecond surface dielectric barrier discharge. Plasma properties in the filaments
}

\author{
S. A. Shcherbanev ${ }^{1}$, Ch. Ding ${ }^{1}$, S. M. Starikovskaia ${ }^{1}$, \\ N. A. Popov ${ }^{2}$ \\ ${ }^{1}$ Laboratory of Plasma Physics (CNRS, Ecole Polytechnique, Univ. Paris-Sud, \\ Observatoire de Paris, Sorbonne Université, l'Institut Polytechnque de Paris), Ecole \\ Polytechnique, route de Saclay, 91128 Palaiseau, France \\ ${ }^{2}$ Skobeltsyn Institute of Nuclear Physics, Moscow State University, Moscow, 119991, \\ Russia \\ E-mail: svetlana.starikovskaia@lpp.polytechnique.fr
}

\begin{abstract}
.
Streamer-to-filament transition is a general feature of nanosecond discharges at elevated pressure. The transition is observed in different discharges by different groups: in the nanosecond surface dielectric barrier discharges (nSDBDs) in a single shot regime at high pressure (2-15 bar), in the point-to-point or point-to-plane open electrodes discharges at high repetitive frequency (so-called nanosecond repetitive pulsed discharges, NRPDs) at atmospherics pressure. The present paper contains experimental analysis of plasma properties in the filamentary nSDBD: the electrical current, the specific deposited energy, the electron density and the electron temperature were measured for a wide range of pressures and voltages. A model explaining plasma properties in filamentary nanosecond discharges and the role of excited species in streamer-to-filament transition is suggested and discussed.
\end{abstract}

Keywords: filamentary discharge, non-equilibrium plasma, nanosecond surface dielectric barrier discharge, nanosecond constriction 


\section{Introduction}

Plasma produced by nanosecond high voltage pulses is widely studied during last 15 years. A broad interest to nanosecond discharges is explained by their potential applications in plasma-assisted combustion [1-3] and detonation [4-6], aerodynamics and flow control [7].

Nanosecond repetitive pulsed discharge (NRPD) [8] was suggested as an efficient tool for plasma-assisted combustion. Different discharge regimes, depending on the gas temperature, the flow rate, the gap between the electrodes and the mean discharge current were studied [9]. Extra-high electron densities, up to $n_{e}=4 \cdot 10^{18} \mathrm{~cm}^{-3}$, were obtained from Stark broadening in pure $\mathrm{N}_{2}$ and in the $\mathrm{N}_{2}: \mathrm{H}_{2} \mathrm{O}$ mixture in nanosecond repetitive pulsed discharge initiated by a $170 \mathrm{~ns} 1 \mathrm{kHz}$ voltage pulse in $2 \mathrm{~mm}$ pin-to-pin gap [10]. The decay of the electron density was slow, a few tens of nanoseconds. Later experimental observations of nanosecond repetitive plasma (NRP) at atmospheric pressure [11-13] also report densities of electrons on the level $n_{e} \sim 10^{18}-10^{19} \mathrm{~cm}^{-3}$, constriction of the discharge channel, high electron temperatures reaching a few electronvolts and slow plasma decay, tens of nanoseconds. The authors of [14] measured the ultra-fast gas heating observed by optical emission spectroscopy on the axis of the streamer before the constriction of the channel.

Independently, the nanosecond surface dielectric barrier discharge (nSDBD) at high pressures was studied in [15-17]. So called streamer-to-filament transition in a single shot experiments in a wide range of pressures [15] and gas mixtures [16,17] was demonstrated. At the moment of transition, during $1-2$ nanoseconds, the emission spectra changed from streamer-like emission containing mainly the molecular bands of $\mathrm{N}_{2}$ to the continuous wavelength $(\mathrm{cw})$ emission with well distinguished broadened lines of atoms $(\mathrm{N}, \mathrm{O}, \mathrm{H})$ and singly ionized atoms, mainly $\mathrm{N}^{+}$. It was shown that the continuous wavelength emission comes from the narrow near-axis zone of the filament. The electron density during the transition changed from $n_{e} \sim 10^{15} \mathrm{~cm}^{-3}$ to $n_{e} \sim 10^{18}-10^{19} \mathrm{~cm}^{-3}$, the decay of the electron density was a few tens of nanoseconds.

The experimental observations prove that both open electrodes NRPD plasma and nanosecond surface DBD plasma in filamentary mode exhibit similar plasma properties. The aim of the present work is (i) to study experimentally the electrical current, the specific deposited energy, the electron density and the electron temperature in the filamentary nanosecond surface barrier discharge at high pressure; (ii) to suggest a model explaining streamer-to-filament in pulsed nanosecond plasma.

\section{Experimental setup}

The coaxial electrode system is described elsewhere [16-18]. A metal disk $20 \mathrm{~mm}$ in diameter served as a high voltage electrode. The internal diameter of the low-voltage grounded electrode was equal to the diameter of the high voltage electrode, and the external diameter of the low voltage electrode was equal to $50 \mathrm{~mm}$. A thin dielectric 


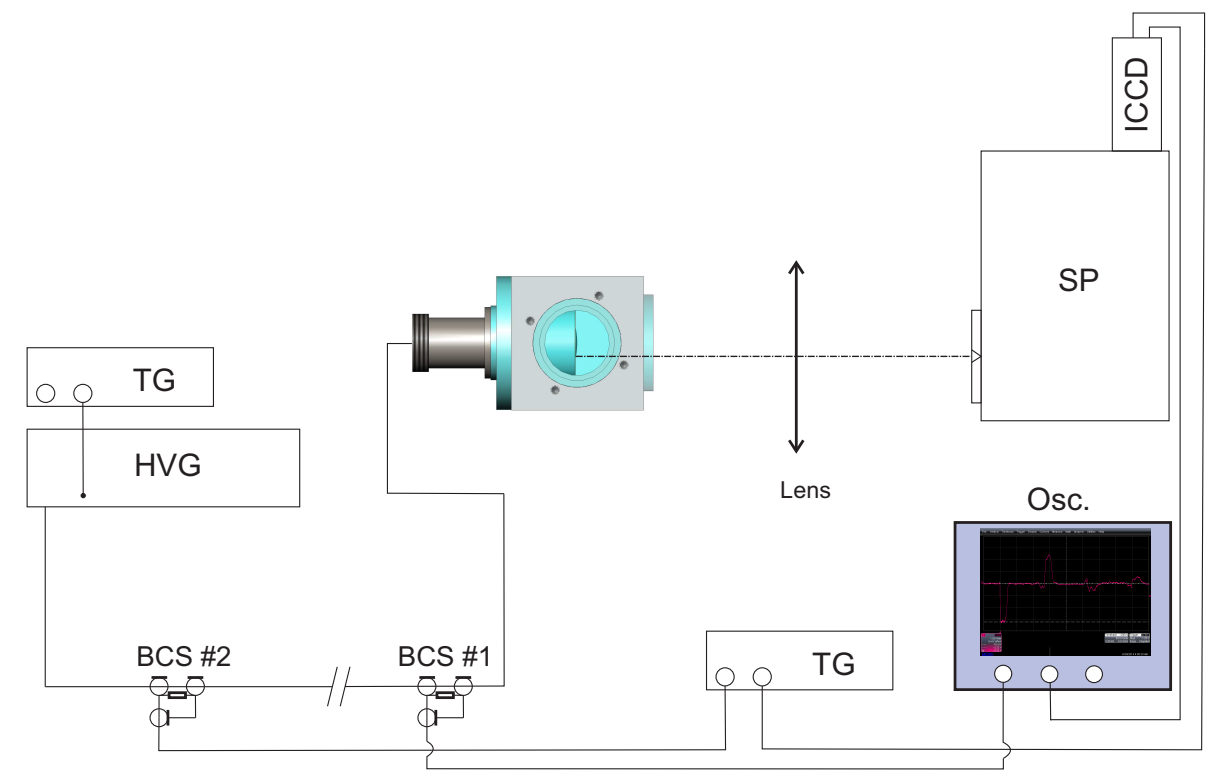

Figure 1. Schematic of the experimental apparatus. SR - spectrograph, ICCD ICCD camera, TG - triggering generator, HVG - high voltage generator, BCS back current shunt, OSC — oscilloscope.

(PVC, $\varepsilon \approx 3-3.5$ ) layer, $0.3 \mathrm{~mm}$ was glued to the grounded electrode by Geocel FIXER Mate silicon glue $(\varepsilon \approx 3)$, the thickness of the glue layer was $0.4 \pm 0.1 \mathrm{~mm}$, giving the total thickness of the dielectric layer equal to $0.7 \pm 0.1 \mathrm{~mm}$. The electrode system was mounted into a constant volume high pressure chamber with 3 quartz optical windows $5 \mathrm{~cm}$ in diameter [17]. The chamber was pumped down to $\sim 10^{-2}-10^{-3}$ Torr before the experiments and then filled with the gas under study at the pressure $P=1-11$ bar. Nitrogen and oxygen (Air Liquide) with $<100 \mathrm{ppm}$ of impurities were used to prepare the mixtures.

All experiments presented in the paper were performed in a single-shot regime. The scheme of synchronization is shown in figure 1 . All electrical signals were registered by LeCroy WaveRunner $600 \mathrm{MHz}$ oscilloscope. ICCD images $(\lambda=300-800 \mathrm{~nm})$ were taken by a Pi-Max4 Princeton Instruments ICCD camera. combined with ACTON 2500i spectrograph. Depending on the resolution and wavelength region of interest three gratings $(600,1200$ and $2400 \mathrm{l} / \mathrm{mm})$ were used.

High-voltage pulse of positive or negative polarity with $2 \mathrm{~ns}$ rise time, $20 \mathrm{~ns}$ duration and $20-60 \mathrm{kV}$ amplitude on the high voltage (HV) electrode (see figure 2(a)) were generated by commercial pulsers FPG20-03PM or FPG20-03NM (FID Technology). The pulse was transmitted to the electrode system via $50 \mathrm{Ohm}$ high voltage coaxial cable. Two calibrated custom made back current shunts (BCS) were installed in the shield of the cable: BCS1 in the middle of the cable, and BCS2 $1 \mathrm{~m}$ apart from the HV pulser. The length of the cable, $30 \mathrm{~m}$, was selected to separate the incident pulse and the pulse, reflected from the discharge cell, on the BCS1 shunt. The BCS1 was used to measure the pulse shape and the absolute value of electrical current. The energy 


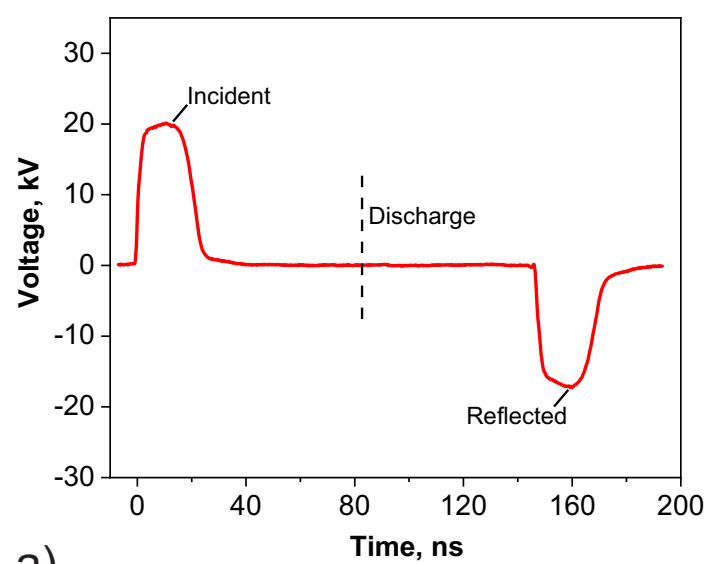

a)

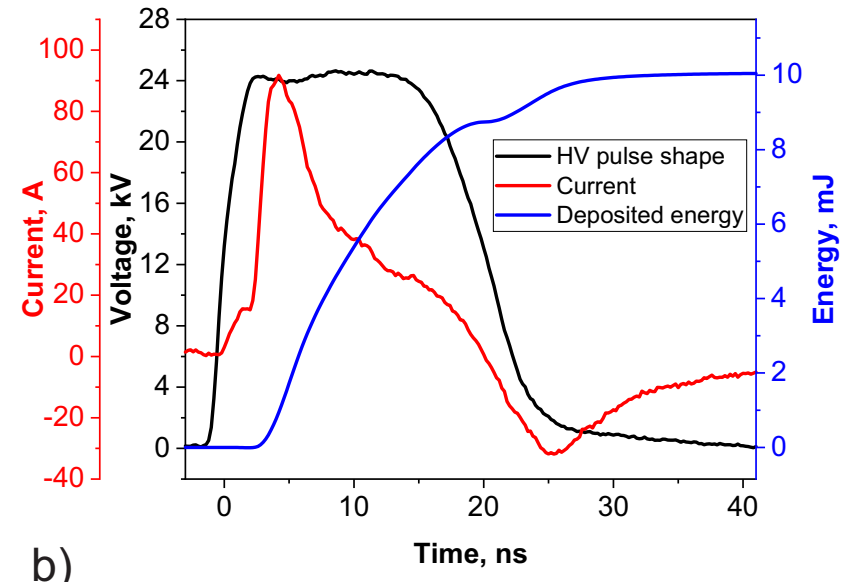

Figure 2. Measured with BCS1: (a) Voltage waveform measured with BCS1, incident and reflected pulses are clearly seen; (b) voltage, current and energy deposition.

deposited into plasma was calculated as the difference between the energies stored in the incident and in the reflected pulses taking into account the energy losses in the cable and the losses on charging of the electrode system. The details of the technique can be found elsewhere [19]. The BCS2 was used to synchronize the ICCD camera with the discharge.

An example of separated in time incident and reflected pulses of voltage in the cable are presented in figure 2 (a). Typical waveforms of voltage, current through the plasma and deposited energy are given by figure2 (b).

\section{Results}

As it was reported previously [15-17], transition to the filamentary mode for both polarities of the high-voltage pulse is characterized by appearance of the continuous spectra in UV and visible ranges, by a sharp increase of electron density up to extremely high values, $n_{e} \sim 10^{18}-10^{19} \mathrm{~cm}^{-3}$, by a long, tens of nanoseconds, decay of electron density, and by intense hydrodynamic perturbations.

\subsection{Electrical current in plasma and ICCD imaging: a basis to derive a specific delivered energy}

Specific delivered energy in filamentary plasma can be estimated on the basis of electrical current measurements combined with ICCD imaging. Figure 3 is a review of typical ICCD images and current waveforms used for the analysis. The ICCD images of a sector of the discharge are presented by figure 3(a); total electrical current through the discharge synchronised with the ICCD images is given by figure 3(b); and finally, the integral ICCD images of the discharge in streamer and filamentary modes are shown in figure 3(c). Here and further "total electrical current" means the summary current through all the channels of the discharge at the conditions that the current of charging of 
the capacitance of the electrode system is subtracted (see section "Experimental setup" for details).

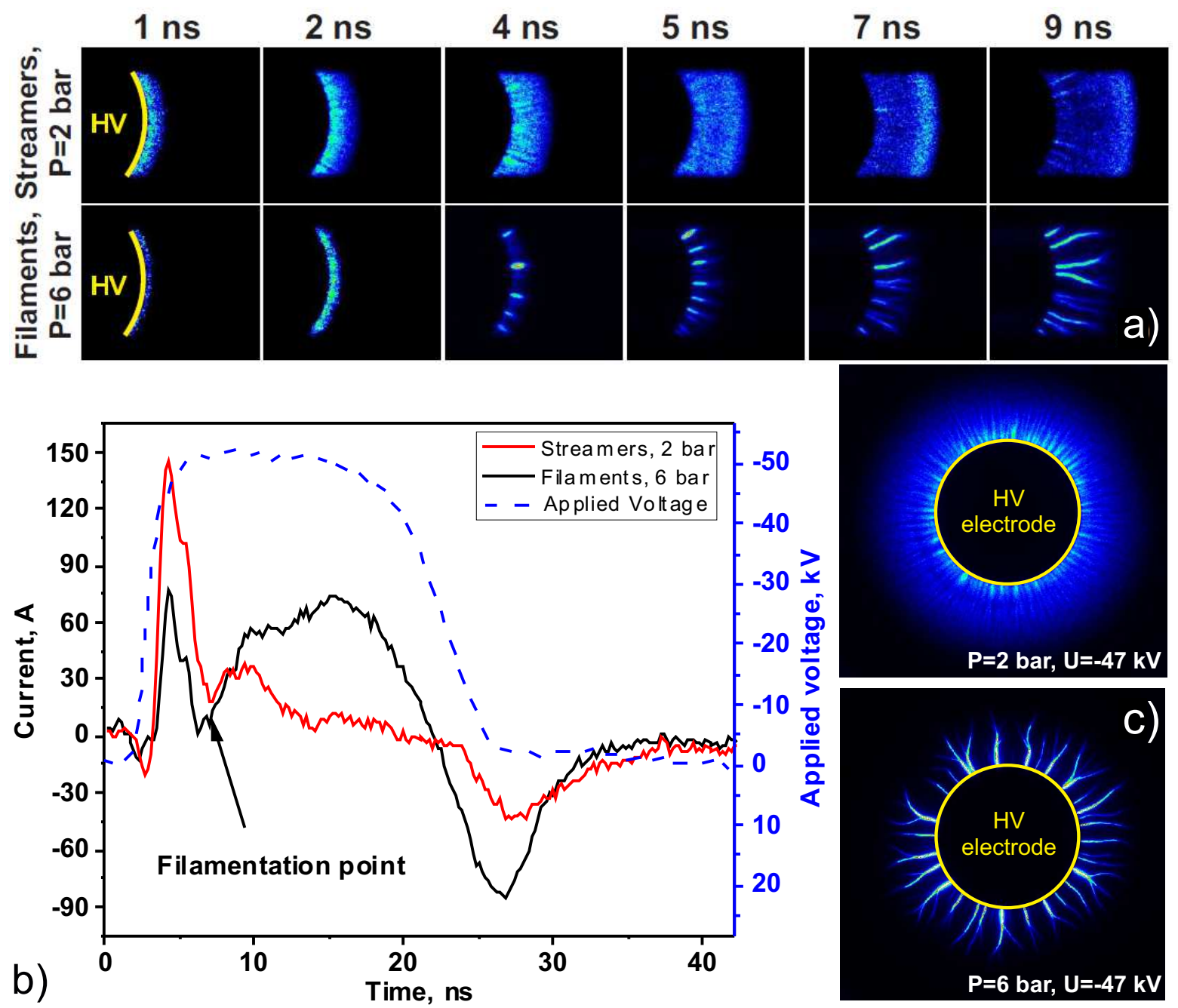

Figure 3. (a) ICCD images of the streamer $(P=2$ bar, $U=-47 \mathrm{kV})$ and filamentary $(P=6$ bar, $U=-47 \mathrm{kV})$ discharges in air. Camera gate is $0.5 \mathrm{~ns}$; (b) Current of streamer and filamentary discharges; (c) Integral ICCD images of the streamer $(P=2$ bar, $U=-47 \mathrm{kV})$ and filamentary $(P=6$ bar, $U=-47 \mathrm{kV})$ discharges in air. Camera gate is $30 \mathrm{~ns}$.

Figure 3(a) compares time resolved ICCD images of streamer and filamentary discharges. At $P=2$ bar discharge develops in the streamer mode. At $P=6$ bar at the time instant $t=4$ ns the filaments (bright channels with high emission intensity) start from the high voltage electrode. When filaments appear (4 ns), the weak glow of the streamers along the perimeter of the HV electrode fills the inter-filamentary space. In the well developed filamentary phase (7 and 9 ns), only bright channels can be distinguished, the intensity of the streamer-like emission in the inter-filamentary space is weak comparing to the emission from filaments. According to [16], the ratio of intensity of emission in the filaments integrated over the sensitivity range of the ICCD camera to the intensity of emission in streamers or between the filaments is around 50 . 
The temporal evolution of the total current is demonstrated in figures 3(b) for streamers at $P=2$ bar and filaments at $P=6$ bar at the same applied voltage $U=-47 \mathrm{kV}$ on the $\mathrm{HV}$ electrode. Waveform of the electrical current of a streamer discharge is described in [20] on the basis of 2D numerical modeling: the first peak of the current $(3-8 \mathrm{~ns}$ in figure $3(\mathrm{~b}))$ is the ion current, the waveform and the value are defined by evolution of the cathode layer taking into account fast charging of the dielectric by electrons [21]; a broad plateau with a small negative slope during the interval of the constant voltage $(8<t<24 \mathrm{~ns})$ corresponding to the formation of the near-surface plasma layer and propagation of the streamer; and the reverse current on the trailing edge of the pulse $(24<t<32 \mathrm{~ns})$ corresponding to the second stroke and to the surface charge elimination. In the first peak $(1-5 \mathrm{~ns})$, the current in the streamer discharge at 2 bar is twice higher than in the discharge at 6 bar, this correlates with the difference in the emission intensity. When filamentation starts, the current increases (black curve) and a few nanoseconds after (7-9 ns after the discharge starts) exceeds the current of streamers (red curve). After 12-15 ns, the current of filaments becomes significantly higher than that of streamers. If the current of streamers is $I_{s t}(12 n s) \simeq 15 \mathrm{~A}$, the current of filamentary discharge is about $I_{f i l}(12 n s) \simeq 60$ A. The current per channel increases due to the fact that the number of filaments at $P=6$ bar is $3-5$ times smaller than the number of streamers at $P=2$ bar. Neglecting the current in the inter-filamentary space, the ratio between the current per filament and the current per streamer is equal to $\left(I_{f i l} / N_{f i l}\right) /\left(I_{s t} / N_{s t}\right) \approx 10$. This ratio is typical for all the observed regimes of streamer-to-filamentary transition: current $0.1 \mathrm{~A} /$ streamer and $1 \mathrm{~A} /$ filament reported in [15] for the transition in $\mathrm{nSDBD}$ in air at 3 bar.

Figure 4(a) compares the total current of the discharge in air at different pressures. Qualitatively, the waveforms of streamer and filamentary discharges are different. At any pressure, the discharge starts as a streamer discharge; the intensity of the first peak decreases with pressure. In the case of the streamer discharge, current decreases monotonously during the plateau of the high-voltage pulse. In the case of the filamentary discharge, at the moment of streamer-to-filamentary transition, the current increases again. For $P=4$ bar, the transition happens at 7 ns; for $P=6$ bar and 8 bar, the transition happens during the first $2-3$ ns.

The energy deposited per discharge channel for two applied voltages $U=-25$ and $-50 \mathrm{kV}$ as a function of gas pressure is shown in figure $4(\mathrm{~b})$. When applied voltage is $U=-25 \mathrm{kV}$, the discharge stays in a streamer mode which appears, for the negative polarity, as a uniform glow at ICCD images. When the voltage amplitude is $U=-50 \mathrm{kV}$, the discharge at pressures 5,6 and 8 bar is filamentary. It is clearly seen that the deposited energy is about 6 times higher for the filamentary discharge. Both in streamer and in filamentary mode, the deposited energy slightly decreases with pressure. As far as the deposited energy is proportional to the product $\mathbf{j} \cdot \mathbf{E}$, and as far as the electron density in the filamentary mode is $4-5$ orders of magnitude higher than in streamers $[16,17]$, we can expect that the electric filed is lower in the filaments.

To analyze the specific deposited energy, namely the deposited energy per molecule, 

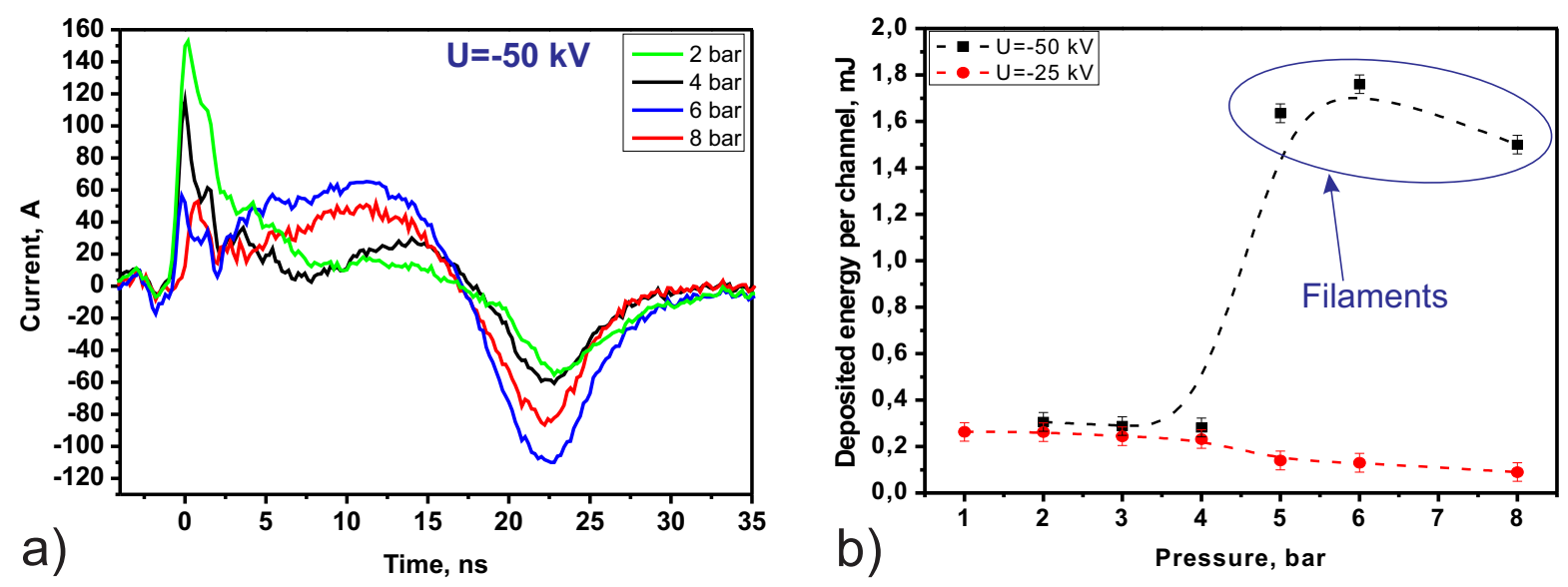

Figure 4. (a) Total current of the discharge in air for different pressures. Applied voltage is $U=-50 \mathrm{kV}$; (b) Energy deposition per discharge channel in air for different pressures. Two cases: $U=-50$ and $-25 \mathrm{kV}$.

it is necessary to estimate the total volume of the filaments. So-called $x-t$ diagrams were used to quantify the propagation of the filaments. To build $x-t$ diagrams, the length of the filaments was analyzed statistically and the length of propagation $x$ at the time instant $t$ was put on the plot.

The velocity of the filaments at constant voltage practically does not depend upon pressure (see figure $5(\mathrm{a})$ ). Different values of the first $x$ points for $P=5,8$ and 11 bar demonstrate that before the transition, streamers cover a longer distance in the case of lower pressure. Being moved at the same initial point, the $x-t$ diagrams for different pressures will follow almost the same way; the velocity of the filament propagation before slowing down is about $0.4 \mathrm{~mm} / \mathrm{ns}$. On the trailing edge of the voltage pulse, at $t>15 \mathrm{~ns}$, the velocity of the filaments decreases. To some extent, it is possible to increase the length of the filaments by increasing the duration of the pulse. A typical length of filaments propagation, comprising $5-10 \mathrm{~mm}$ at pressures up to ten bars, is an interesting issue for plasma-assisted combustion: it was mentioned in [18] that the filamentary regime, providing a regular grid of tens of plasma channels with high temperature and high density of $\mathrm{O}$-atoms, results in efficient and stable ignition of combustible mixtures. Longer filaments will result in larger region of ignition.

When the pressure is fixed and the voltage amplitude is changed (see figure $5(\mathrm{~b})$ ), the velocity of filaments is not constant any more: increase of the voltage amplitude from $U=-35 \mathrm{kV}$ to $U=-42 \mathrm{kV}$ leads to acceleration of the filaments from $0.3 \mathrm{~mm} / \mathrm{ns}$ to $0.5 \mathrm{~mm} / \mathrm{ns}$. The regime with pronounced difference in the velocity of filaments, constant pressure $P=8$ bar and the voltage amplitude changing between $U=-33 \mathrm{kV}$ and $U=-43 \mathrm{kV}$ was selected to study the specific deposited energy.

A maximum length of the filaments propagation at different voltage amplitudes is presented in figure 6 (a). The length increases significantly, from $2.7 \mathrm{~mm}$ at $U=-33 \mathrm{kV}$ to $10 \mathrm{~mm}$ at $U=-43 \mathrm{kV}$. The total deposited energy measured from the waveforms obtained by a back current shunt, also increases with voltage, from $5.9 \mathrm{~mJ}$ at 

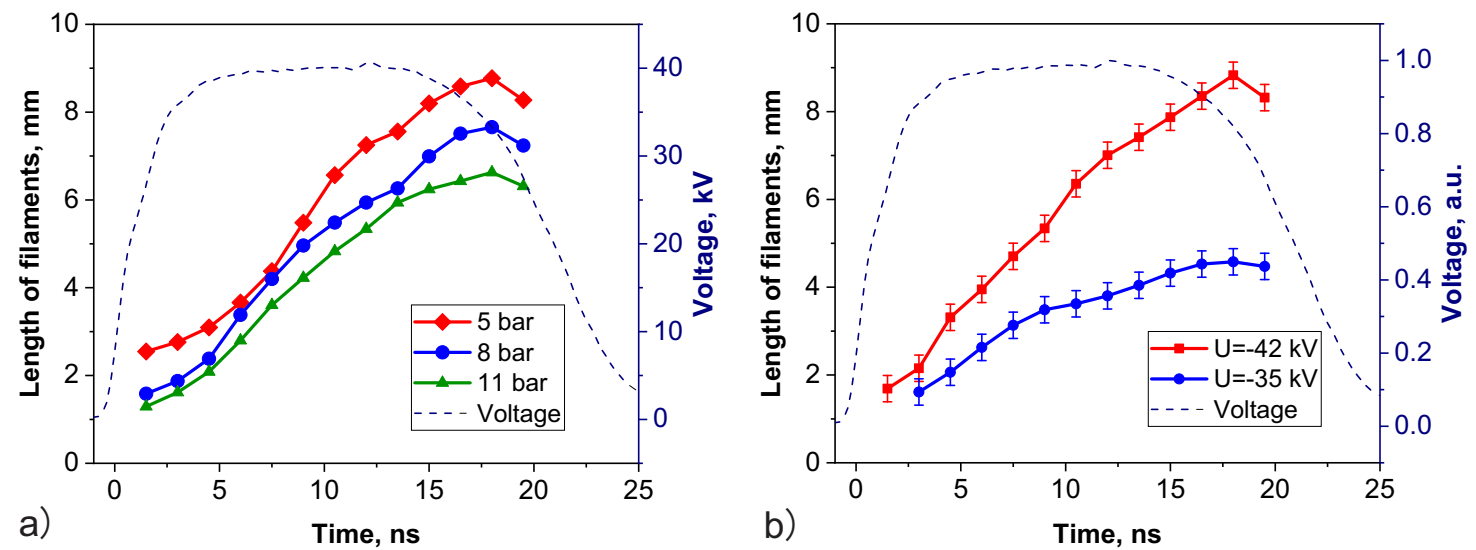

Figure 5. x-t diagrams of propagation of filaments for discharge in air at negative polarity: (a) for different pressures; the applied voltage amplitude is $U=-40 \mathrm{kV}$; (b) for different voltages; the pressure is $P=8$ bar.
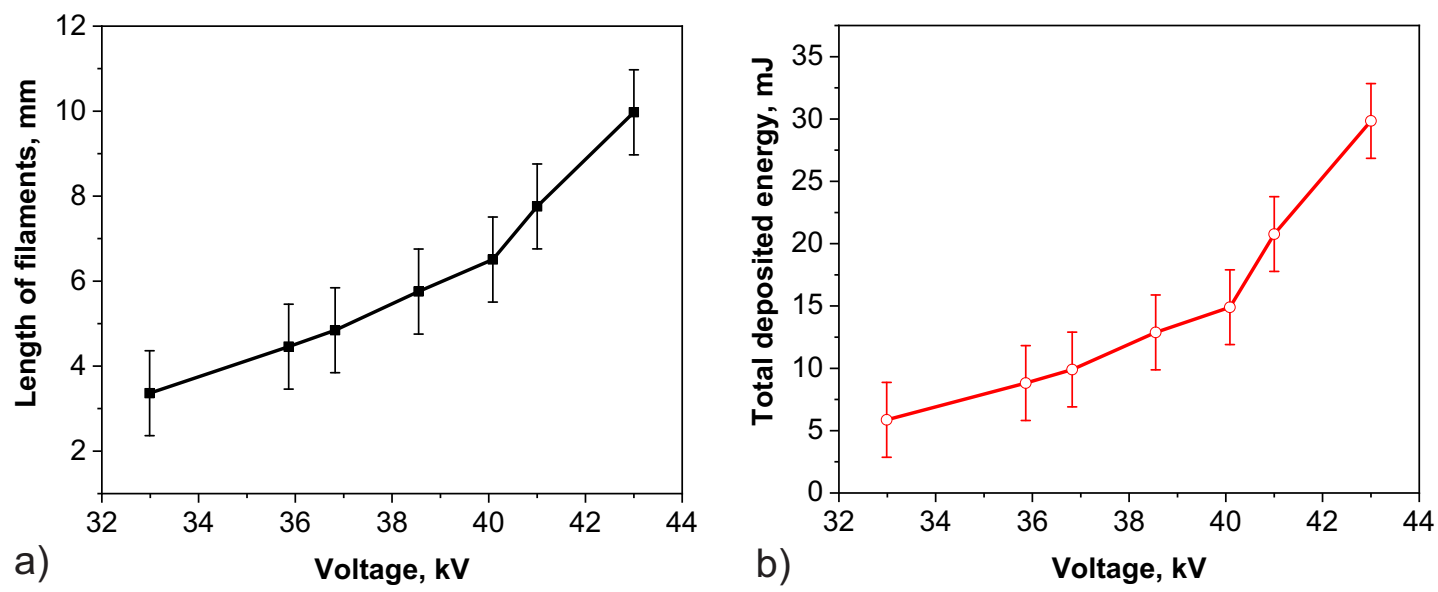

Figure 6. A maximum length of the filaments propagation (a) and the total deposited energy (b) as a function of the voltage amplitude. Air, negative polarity of the pulse, $P=8$ bar.

$U=-33 \mathrm{kV}$ to almost $30 \mathrm{~mJ}$ at $U=-43 \mathrm{kV}$ (figure $6(\mathrm{~b})$ ). The last parameter to be taken into account is the number of filaments increasing from 30 at $U=-33 \mathrm{kV}$ to 47 at $U=-43 \mathrm{kV}$ (figure 7 (a)). Assuming that the energy is equally distributed between the filaments, will obtain that the energy changes from 0.20 to $0.64 \mathrm{~mJ}$ per filament with voltage. Finally, assuming that the energy is distributed uniformly along the filament, and dividing the energy per filament by the filament length, will get the plot presented in figure 7 (b). It is clearly seen that in spite of strong dependence of parameters on voltage, the energy per unit length is almost constant for all voltage amplitudes under study.

To determine the specific deposited energy, it is necessary to know the cross section of the filament. The upper limit of the diameter of the filament can be derived from the filtered ICCD macroimages presented in figure 8. The images were taken (from left to 

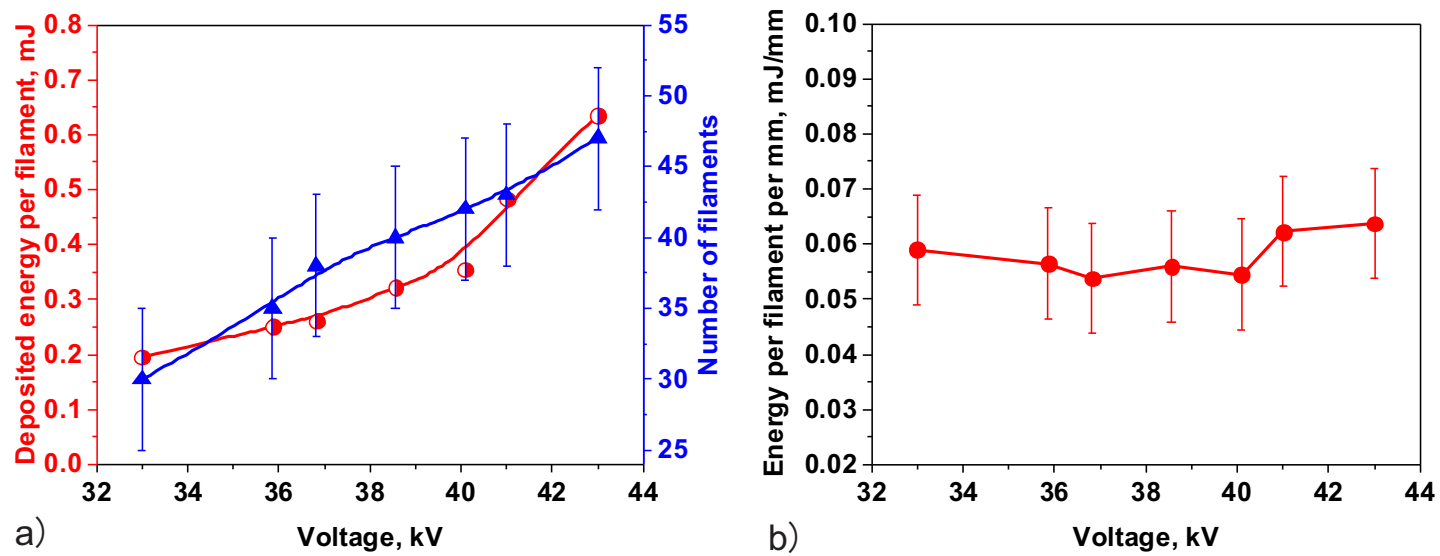

Figure 7. Number of filaments and the deposited energy per filament (a); the energy per filament per $\mathrm{mm}$ (b) as a function of the voltage amplitude. Air, negative polarity of the pulse, $P=8$ bar.

right) without a filter; with $480 \pm 5 \mathrm{~nm}$ filter selecting a spectral region with no molecular bands of $\mathrm{N}_{2}$; and with $340 \pm 5 \mathrm{~nm}$ filter selecting the second positive system of $\mathrm{N}_{2},(0-0)$ vibrational transition at $\lambda=337.1 \mathrm{~nm}$. It is clearly seen that the filament captured with no filter can be represented as a thin bright core along the axis of the filament; the core is surrounded by a less bright shell. The diameter of the core calculated as the FWHM of the emission at $480 \pm 5 \mathrm{~nm}$ is equal to $18 \pm 2 \mu \mathrm{m}$ while the diameter of the entire structure is around $200-300 \mu \mathrm{m}$. The bright core is a region where from the $c w$ radiation is observed $[16,17]$. In assumption that almost all the delivered energy is spent in the core of the filament, $18 \pm 2 \mu \mathrm{m}$ diameter, will estimate the energy per molecule at taking the number of molecules to be equal to the number of molecules at $P=8$ bar and $T=300 \mathrm{~K}$. For the energy per unit length $\sim 0.06 \mathrm{~mJ} / \mathrm{mm}$ as obtained for the conditions of the present work figure 7 (b), will obtain the specific delivered energy $\omega \approx 7.4 \mathrm{eV} /$ particle.

\subsection{Synchronized electron temperature and electron density measurements}

To understand the behavior of the electron temperature in the filamentary discharge and in the near afterglow, the emission of atomic nitrogen ions in the wavelength range $\lambda=480-600 \mathrm{~nm}$ was analyzed. The experiments were performed in the positive polarity discharge in nitrogen at the pulse amplitude $U=47 \mathrm{kV}$ on the high-voltage electrode. The data were taken with the ICCD camera gate equal to 5 ns. The raw spectra were corrected to the spectral sensitivity of the system; the background corresponding to the continuous wavelength emission was subtracted. Treated in the described way spectra were modeled using NIST database [22] and taking the electron temperature $T_{e}$ as a parameter. A few examples of experimentally obtained and calculated spectra are presented in figure 9. A good correlation between the experiment and modeling is clearly seen.

The electron density was calculated in the afterglow on the basis of the Stark 


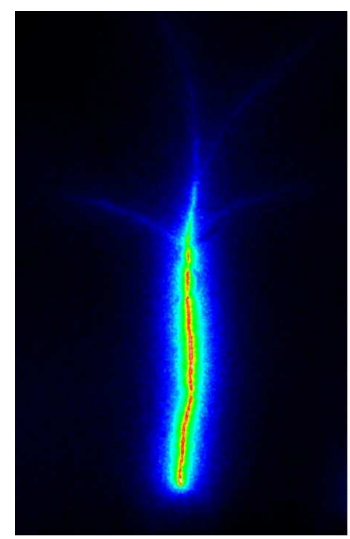

No filter

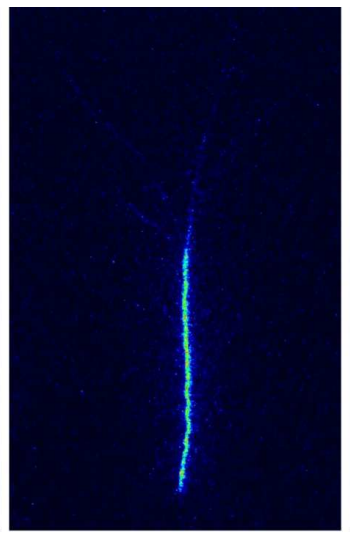

$480 \mathrm{~nm}$ filter

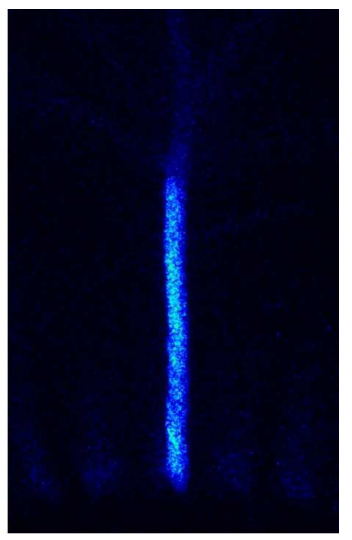

$340 \mathrm{~nm}$ filter

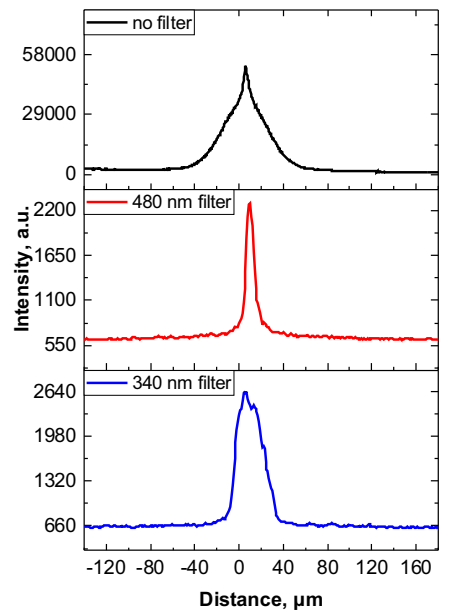

Figure 8. Macro-images of the filamentary nSDBD without narrow band filters and with $480 \pm 5 \mathrm{~nm}, 340 \pm 5 \mathrm{~nm}$ filters. Positive polarity, $U=45 \mathrm{kV}$, air, $P=8$ bar.
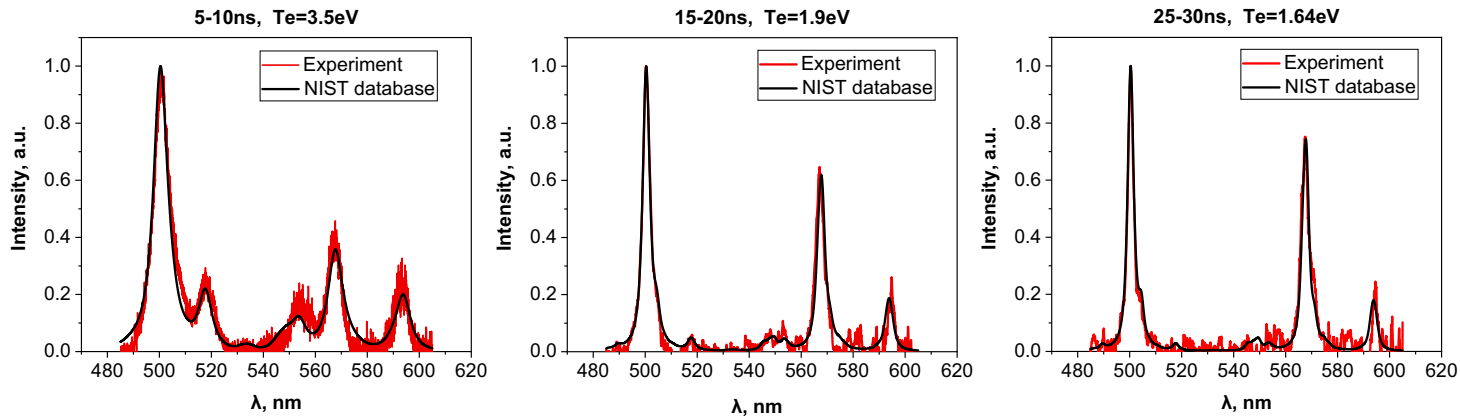

Figure 9. Emission lines of $\mathrm{N}^{+}$ions.

broadening of the $\mathrm{H}_{\alpha}$ line $(\lambda=656.3 \mathrm{~nm})$ and of the $\mathrm{N}$-atom lime $(\lambda=746.8 \mathrm{~nm})$ taking into account the pressure broadening for $\mathrm{N}$-atoms, the details of the technique can be found elsewhere [10].

Synchronized waveforms of voltage on the electrode, electrical current through the discharge, electron temperature and electron density are presented in figure 10. The accuracy of the synchronization, provided by the BCS technique, is $0.5 \mathrm{~ns}$. Streamerto-filament transition happens at $t \approx 7 \mathrm{~ns}$. The electron temperature in the beginning of the filamentary phase is $T_{e}=3-3.5 \mathrm{eV}$, falling down to $T_{e}=1.6-1.7 \mathrm{eV}$ during the discharge and staying at this level at least first $10 \mathrm{~ns}$ of the afterglow. The electron density in these experiments was measured in the afterglow only. It drops slowly when the discharge is off, starting from $n_{e} \approx 5 \cdot 10^{18} \mathrm{~cm}^{-3}$ immediately after the discharge and decreasing to $n_{e} \approx 6 \cdot 10^{17} \mathrm{~cm}^{-3} 40 \mathrm{~ns}$ later. 


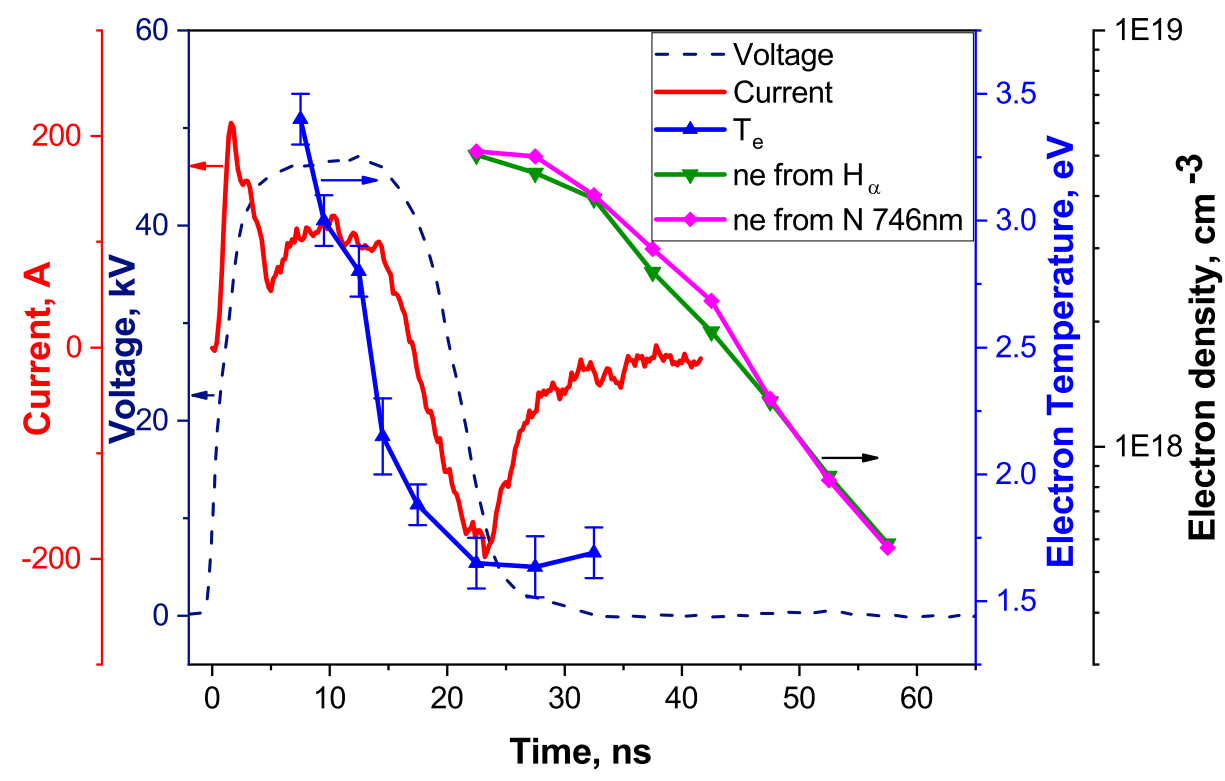

Figure 10. Synchronized electron density and temperature measurements. Discharge in nitrogen at positive polarity of the high-voltage pulse, $U=47 \mathrm{kV}$.

\section{Discussion. Mechanism of discharge constriction at high pressures in the nanosecond time scale}

\subsection{Properties of plasma in the filaments}

According to experimental observations in different gas mixtures (figure 10 for the afterglow, [17] for the discharge and the afterglow), high values of electron density, $n_{e}=10^{18}-10^{19} \mathrm{~cm}^{-3}$ exist during at least $5-10 \mathrm{~ns}$. Based on this data, let us estimate the heat release in the reactions of electron-ion recombination. The main ions responsible for recombination at sub-nanosecond times scale are molecular ions $\mathrm{N}_{2}^{+}$and

$\mathrm{O}_{2}^{+}$. Until the fraction of the molecules in the gas is low enough, recombination happens mainly in the reactions

$$
\begin{aligned}
& \mathrm{e}+\mathrm{O}_{2}^{+} \rightarrow \mathrm{O}\left({ }^{3} \mathrm{P}\right)+\mathrm{O}\left({ }^{1} \mathrm{D}\right) \\
& \mathrm{e}+\mathrm{N}_{2}^{+} \rightarrow \mathrm{N}\left({ }^{4} \mathrm{~S}\right)+\mathrm{N}\left({ }^{2} \mathrm{D}\right)
\end{aligned}
$$

The recombination is fast, a typical time of the processes (R1) and (R2) at $n_{e}=2 \cdot 10^{18} \mathrm{~cm}^{-3}$ is $\tau_{\text {rec }} \approx 0.4 \mathrm{~ns}$. Each elementary process results in severe heat release: $5 \mathrm{eV}$ in the reaction $(\mathrm{R} 1)$ and $2.25 \mathrm{eV}$ in the reaction (R2) respectively [23]; and the additional heat will be produced due to the quenching of excited $\mathrm{O}\left({ }^{1} \mathrm{D}\right)$ and $\mathrm{N}\left({ }^{2} \mathrm{D}\right)$ atoms. Only due to the heat release in the reactions (R1) and (R2) the gas temperature during less than $1 \mathrm{~ns}$ increases up to $15-20 \mathrm{kK}$. Thus the consequence of a fast recombination at high electron density, on the level of $n_{e} \geq 2 \cdot 10^{18} \mathrm{~cm}^{-3}$, is increase of gas temperature and approaching of plasma to the local thermodynamic equilibrium 
(LTE), with the gas temperature close to the electron temperature.

The electron-ion collision frequency $\nu_{e i}$ increases with the electron density [24]:

$$
\nu_{e i}=\frac{n_{e} \cdot e^{4} \cdot \ln \Lambda}{18 \sqrt{2} \pi \cdot \varepsilon_{0}^{2} \cdot \sqrt{m_{e}} \cdot\left(T_{e}\right)^{3 / 2}},
$$

Here $\ln \Lambda$ is the Coulomb logarithm, $m_{e}$ and $e$ are the mass and the charge of the electron respectively, $T_{e}$ is the electron temperature, $\varepsilon_{0}$ is the dielectric constant.

At the conditions that plasma is strongly ionized and the electron-ion collisions dominate, the conductivity is calculated as

$$
\sigma_{e i}=\frac{n_{e} \cdot e^{2}}{m_{e} \cdot \nu_{e i}}
$$

For a typical electron density $n_{e}=3 \cdot 10^{18} \mathrm{~cm}^{-3}$ and electron temperature $T_{e}=3 \mathrm{eV}$, the value of the Coulomb logarithm is $\ln \Lambda=5.05$, and so the conductivity $\sigma_{e i} \approx$ $180 \mathrm{Ohm}^{-1} \cdot \mathrm{cm}^{-1}[24]$. In this case, for the electric current density $j_{f} \leq 3 \cdot 10^{5} \mathrm{~A} / \mathrm{cm}^{2}$, the electric field in the filaments does not exceed $E_{f}=1700 \mathrm{~V} / \mathrm{cm}$. Such a low field in the constricted channels is the main reason for the sharp decrease in the emission intensity of the $2^{+}$nitrogen system, which is observed in nSDBD during the streamerto-filament transition [16,17]. It should be noted that as far as the field in the filaments is low, their propagation length is defined by the duration of the pulse (see figure 5 (a)), and so, using longer pulses, it is possible to obtain longer filament length.

The equilibrium electron density calculated as a function of gas temperature is presented in figure 11 for two cases: pressure $P=1$ bar and initial gas number density $N_{0}=2.5 \cdot 10^{19} \mathrm{~cm}^{-3}$; pressure $P=5$ bar and initial gas number density $N_{0}=1.2 \cdot 10^{20} \mathrm{~cm}^{-3}$. The calculations were carried out using the model [26] in isochoric approximation. The approximation is valid because the time of filamentation, a few nanoseconds, is much shorter than a typical hydrodynamic time; the gas expansion does not occur and the particle density remains approximately constant, increasing only due to the dissociation of mixture molecules. It follows from the figure that at $P=5$ atm, the equilibrium electron density $n_{e}=(2-3) \cdot 10^{18} \mathrm{~cm}^{-3}$ is reached at a gas temperature equal to $T_{g}=13 \pm 0.6 \mathrm{kK}$.

Decay of the electron density from $n_{e}=5 \cdot 10^{18} \mathrm{~cm}^{-3}$ to $n_{e}=6 \cdot 10^{17} \mathrm{~cm}^{-3}$ during a few tens of nanoseconds at $P=5$ bar can be explained by gas cooling from $T_{g}=14 \mathrm{kK}$ to $T_{g}=10 \mathrm{kK}$. Estimates of gas cooling via thermal conductivity using a coefficient of heat conductivity taken from [24] result in typical time tens of nanoseconds at a typical radius of channel equal to $10 \mu \mathrm{m}$. It should be noted that the cooling associated with the gas-dynamic expansion of the channel was not taken into account in the estimates.

Let us consider the processes that allow obtaining the high values of electron density, reached experimentally, $n_{e}>10^{18} \mathrm{~cm}^{-3}$ at $P=5-6$ atm during a sub-nanosecond time. Figure 12 presents calculated kinetic curves of the main components and the evolution of the gas temperature in molecular nitrogen at initial pressure $P_{0}=6$ bar, for the initial gas temperature $T_{0}=300 \mathrm{~K}$ and constant reduced electric field $E / N=230 \mathrm{Td}$, which corresponds to the measured electron temperature in the discharge, $T_{e}=3-3.5 \mathrm{eV}$. 


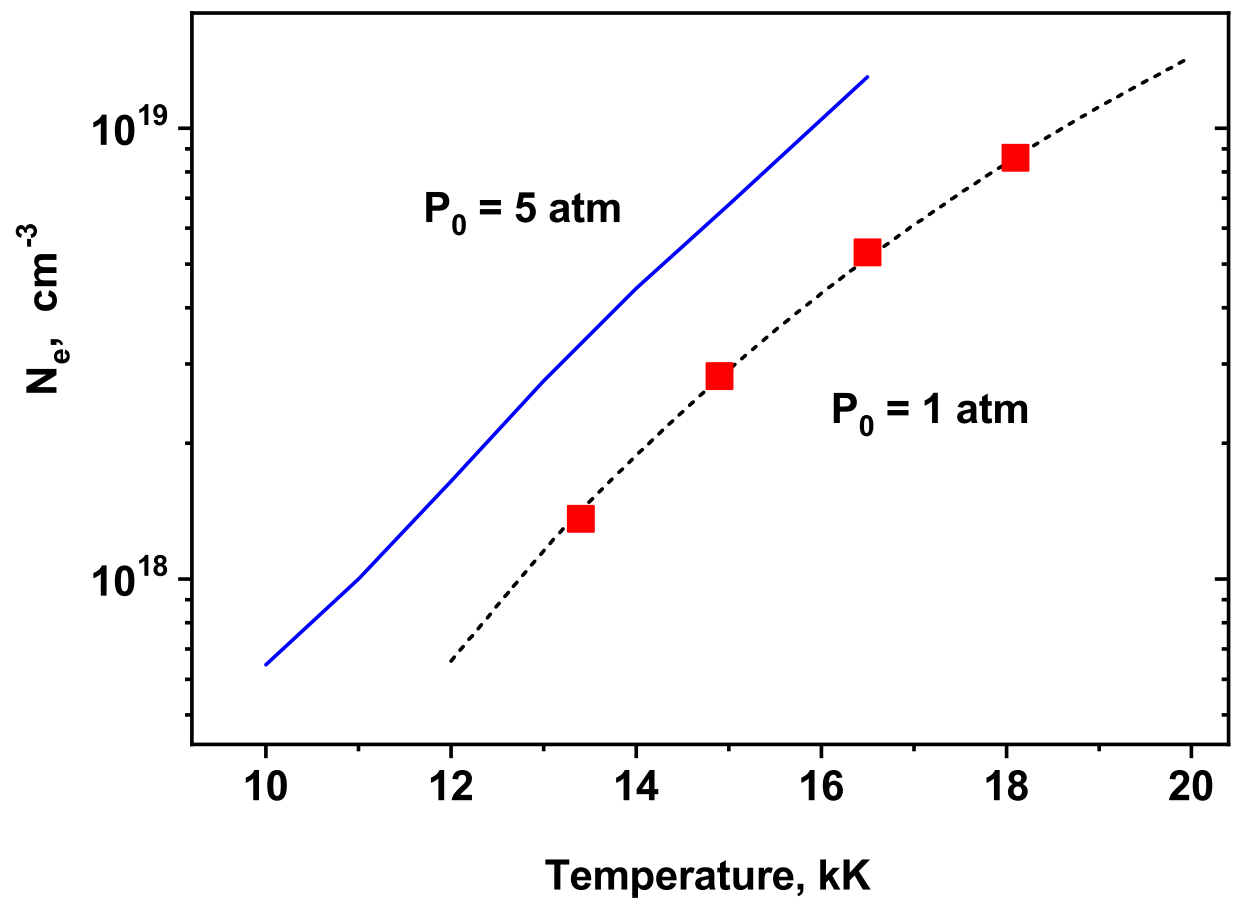

Figure 11. Calculated electron density as a function of gas temperature for $P=1$ bar at the initial gas number density $N_{0}=2.5 \cdot 10^{19} \mathrm{~cm}^{-3}$, and for $P=5$ bar at the initial gas number density. $N_{0}=1.2 \cdot 10^{20} \mathrm{~cm}^{-3}$. Symbols are the experimental data from [25].

The calculations were carried out in isochoric approximation; rates of ionization and excitation of nitrogen atoms and molecules by electron impact were calculated using BOLSIG+ software [27]. The self-consistent sets of the cross-sections for elastic and inelastic electron collisions were taken from [28,29] for $\mathrm{N}_{2}$ molecules and from [30] for nitrogen atoms. The cross-sections of ionization and dissociation of $\mathrm{N}_{2}\left(\mathrm{~A}^{3} \Sigma_{u}^{+}, \mathrm{B}^{3} \Pi_{g}\right.$, $\mathrm{C}^{3} \Pi_{u}$ ) molecules by electron impact, as well as cross-sections of the transitions between these states were taken from [31].

At short time period, $t<0.15 \mathrm{~ns}$, the density of electronically excited nitrogen molecules, $\mathrm{N}_{2}\left(\mathrm{~B}^{3} \Pi_{g}, \mathrm{C}^{3} \Pi_{u}\right)$ increases proportionally to the density of electrons. At $n_{e}>3 \cdot 10^{16} \mathrm{~cm}^{-3}$, quenching of electronically excited $\mathrm{N}_{2}\left(\mathrm{~A}^{3} \Sigma_{u}^{+}, \mathrm{B}^{3} \Pi_{g}, \mathrm{C}^{3} \Pi_{u}\right)$ states by electrons dominates above quenching by nitrogen molecules. A significant part of dissociation at these conditions goes via dissociation of electronically excited states by electron impact:

$$
\mathrm{e}+\mathrm{N}_{2}\left(\mathrm{~A}^{3} \Sigma_{\mathrm{u}}^{+}, \mathrm{B}^{3} \Pi_{\mathrm{g}}, \mathrm{C}^{3} \Pi_{\mathrm{u}}\right) \rightarrow \mathrm{e}+\mathrm{N}\left({ }^{4} \mathrm{~S}\right)+\mathrm{N}\left({ }^{2} \mathrm{D}\right)
$$

Similar, ionization of electronically excited states by electron impact is important under the present conditions:

$$
\mathrm{e}+\mathrm{N}_{2}\left(\mathrm{~B}^{3} \Pi_{g}, \mathrm{C}^{3} \Pi_{u}\right) \rightarrow 2 \mathrm{e}+\mathrm{N}_{2}^{+}\left(\mathrm{X}^{2} \Sigma_{g}^{+}, \mathrm{A}^{2} \Pi_{u}\right) .
$$

At $E / N=230 \mathrm{Td}$, ionization from excited states is equal to ionization from the ground state starting from $\left[\mathrm{N}_{2}\left(\mathrm{~B}^{3} \Pi_{g}, \mathrm{C}^{3} \Pi_{u}\right)\right] /\left[\mathrm{N}_{2}\right] \geq 10^{-3}$. As a result, already 
at $t>0.15 \mathrm{~ns}$, the main ionization takes place from the electronically excited levels. To underline the role of ionization from the excited states at high electric field and high deposited energy, the dashed curve in figure 12 presents the electron density calculated without the reaction (R4). It is clearly seen that the electron density calculated with the stepwise ionization (R4) at $t=0.35 \mathrm{~ns}$ is about 15 times higher than the electron density obtained via ionization from the ground state only.

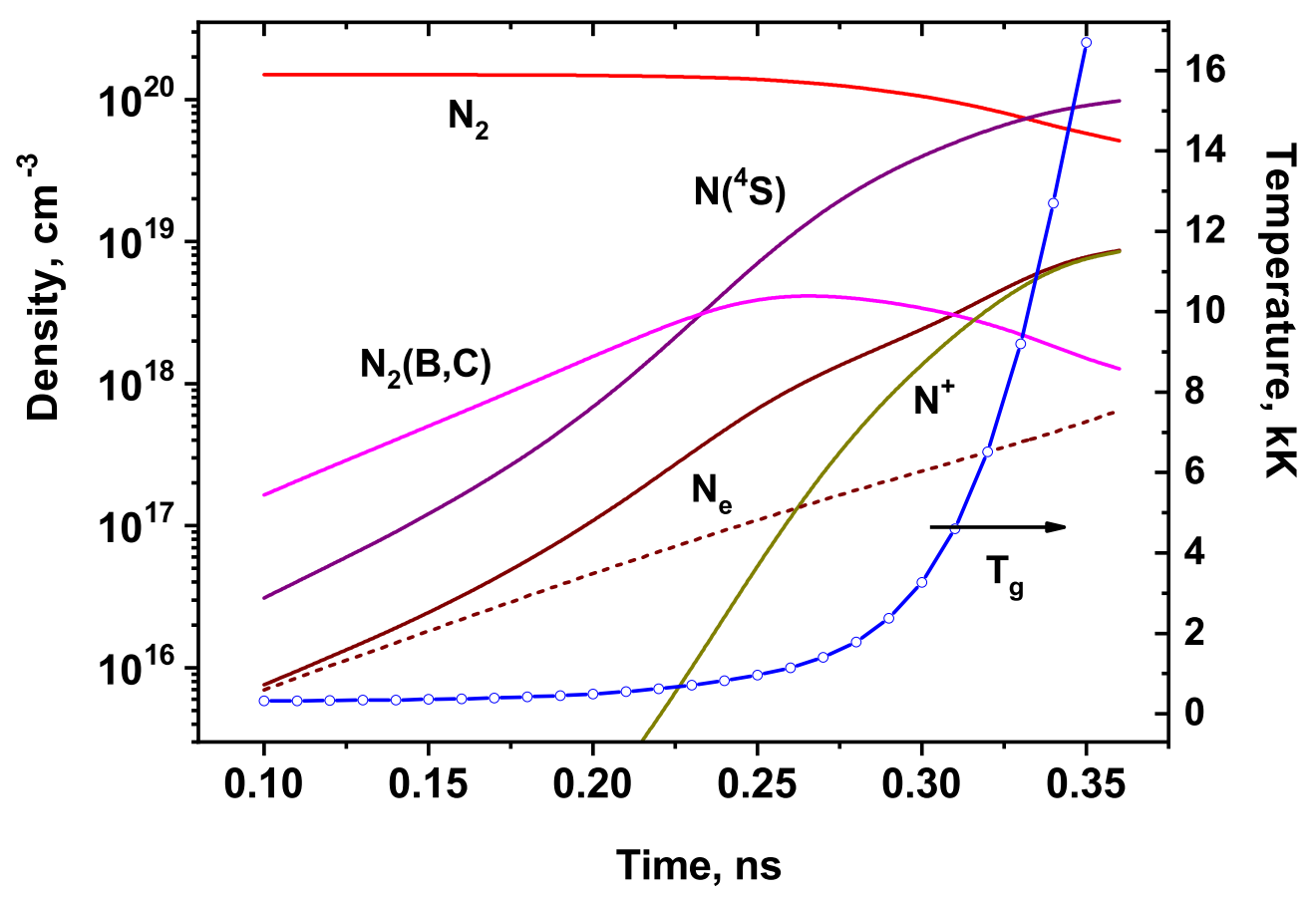

Figure 12. Calculated kinetic curves of main species and gas temperature in discharge in nitrogen at $P=6$ bar, $T_{0}=300 \mathrm{~K}, E / N=230 \mathrm{Td}$.

Gas temperature presented in figure 12 increase dramatically at sub-nanosecond time scale. The temperature was calculated under the assumption that the difference between the specific delivered energy at the given time instant, $\omega(t)$, and the specific energies spent for dissociation, $\omega_{\text {diss }}$, ionization, $\omega_{i o n}$ and electronic excitation of atoms and molecules, $\omega_{\text {exc }}, \Delta \omega=\omega-\omega_{\text {diss }}-\omega_{\text {ion }}-\omega_{\text {exc }}$, instantaneously transforms into the heat. Necessary thermodynamic functions were taken from [32].

The ratio of the frequency of electron-ion collisions to the frequency of electronneutral collisions, $\nu_{e i} / \nu_{e n}$, increases with the ionization degree, $n_{e} /\left[\mathrm{N}_{2}\right]$. As a result, the electron temperature, and the rates of gas ionization and excitation decrease. At $t>0.28 \mathrm{~ns}$ the rates of increase of electron density and of nitrogen atoms density slow down. The electron density at $t=0.33 \mathrm{~ns}$ is equal to $n_{e}=5.3 \cdot 10^{18} \mathrm{~cm}^{-3}$, the density of nitrogen atoms is equal to $\left[\mathrm{N}\left({ }^{4} \mathrm{~S}\right)\right] \approx 7 \cdot 10^{19} \mathrm{~cm}^{-3}$, and the gas temperature is equal to $T_{g} \approx 12-14 \mathrm{kK}$. At this time instant, the specific delivered energy is equal to $\omega \approx 8.8 \mathrm{eV} /$ molecule. Later in time, the system will approach to the equilibrium state 
at corresponding gas temperature.

To summarize, the high electron density in the beginning of the filamentation, appearing in reactions of ionization from electronically excited states, leads to significant increase in electric current density. This is the result of the discharge constriction, leading to an increase in the specific delivered energy and heat release on the time scale of parts of nanoseconds. Approaching to the state close to the LTE explains the anomalously slow plasma decay, tens of nanoseconds, observed experimentally: the decay of electron density follows the dynamics of the gas cooling.

It should be noted that in a number of works $[33,34]$, it is assumed that the discharge constriction is the result of magnetic compression of plasma channel. The magnetic pressure $P_{m}$ is a function of the discharge current $I$ and of the filament radius $R_{f}$ :

$$
P_{m}=\frac{10^{-7} \cdot \mu I^{2}}{16 \pi^{2} R_{f}^{2}} .
$$

Will demand that the magnetic pressure $P_{m}$ is equal to the gas pressure $P_{g}$ inside the filament. In assumption that at $P_{0}=6$ atm the gas temperature will reach the value of $15 \mathrm{kK}$ during parts of nanosecond (see figure 12), the gas pressure in the channel on this stage is equal to $300 \mathrm{~atm}$. At discharge current $1 \mathrm{~A}$ per filament, the magnetic pressure $P_{m}$ will be equal to gas pressure $P_{g}$ for the radius of filaments as small as $R_{f}<0.015 \mu \mathrm{m}$. Thus, the magnetic compression is not important under conditions of the nanosecond surface discharge.

To answer the question of initiation of the filament, the near-electrode processes should be considered, for example development of the field emission instability of the cathode layer [35]. At high pressures, this instability should be efficient because of high value of the electric field near the cathode, which leads to significant decrease of the cathode work function [24] and increase of the secondary electron emission current.

\subsection{Near-electrode processes and filament propagation as an ionization wave}

Recent numerical calculations of a cathode layer in a pulsed nanosecond discharge in helium at high pressure [36] demonstrated a possibility of a high electron density generation near the cathode. The calculations were carried out using $1 \mathrm{D}$ self-consistent Particle-in-Cell Monte Carlo collisions model. High densities of electrons, $n_{e}>$ $10^{18} \mathrm{~cm}^{-3}$, were obtained as a result of secondary electron emission from the cathode surface. The account of field emission resulted in shorter time of formation of the cathode layer. Under the conditions of [36], the electron density reached a maximum value $n_{e}^{\max }$ at $t<2 \mathrm{~ns}$, and the current density of the field emission was as high as $j_{f e}>10^{8} \mathrm{~A} / \mathrm{cm}^{2}$.

In [33] the initial stage of nanosecond discharge at atmospheric pressure was studied with extra-high spatial (3-4 $\mu \mathrm{m})$ and temporal $(70 \mathrm{ps})$ resolution. The authors demonstrated, that nanosecond breakdown initiated by 4 ns rise time $25 \mathrm{kV}$ amplitude voltage pulse in $3 \mathrm{~mm}$ pin-to-plane gap starts from a dense spot of electrons near the cathode, with a density $n_{e}^{0}>10^{20} \mathrm{~cm}^{-3}$ and a typical size of $\sim 10 \mu \mathrm{m}$. The authors [33] 
remark that the nature of the observed electron cloud is still under discussion and mention the field emission in strong electric field and a possibility of production of a supercritical fluid during explosive heating of a single spike or multiple spikes on the cathode.

Non-numerous experimental observations $[11,13,14,37]$ obtained in mm or sub-mm pin-to-pin gaps report the start of the filament from the cathode, then, with a delay of the order of $1 \mathrm{~ns}$ - from the anode, and then the filaments merge in the middle of the gap $[13,37]$. Significant decrease of the streamer radius, by a factor of $3-5$, on the time scale $0.5 \mathrm{~ns}$ before the filamentation is recently reported [13]. Significant changes of parameters of the streamer plasma in the radial direction before the filamentation were observed in [14]: significant increase of gas temperature in the middle of the channel was measured; significant, 5-6 times, decrease of radius was reported.

In nanosecond surface dielectric barrier discharges, for both polarities of the highvoltage electrode the filaments start from the edge of the HV electrode and propagate to the bulk of the streamer plasma. In the case of the cathode a reason of formation of the high electron density near the electrode can be a field emission instability, similar to the case described in [36]. A question about the instability of the anode layer and possible formation of anode spots in high pressure SDBD remains open. The model suggested in the present paper assumes that the region with a high electron density is formed near the HV electrode. The potential on the front of this region is significantly higher than the potential of the streamer plasma at this point. The sharp gradient of electrical potential near the electrode initiates the wave of a gradient of the electrical potential. As a result, the surface streamers formed at the head of filaments [16] additionally charge the surface of the dielectric.

It should be noted that in nSDBDs, unlike to pin-to-pin electrode systems, the density of the electrical current increases not only because of contraction: the additional increase is connected to decrease of the number of plasma channels at the moment of the streamer-to-filament transition. In abnormal mode of the discharge, increase of the current density in the microchannels near the cathode results in rise of the electric field near the cathode, $E_{c}$. High electric fields $E_{c}$ lead to increase of the current density due to the field emission and to development the instability of cathode layer.

It is observed experimentally that in nanosecond surface dielectric barrier discharge, the filaments formed near the high-voltage electrode propagate to the periphery of the discharge with a typical velocity of a parts of millimeters per nanosecond, and the velocity is almost independent of the length of the filament. According to [38], so high propagation velocities are typical for so-called fast ionization waves (FIW) or the waves of a gradient of the electrical potential. Will estimate a possible speed of FIW under the considered conditions. A necessary condition for the existence of FIW is that the formation of the next element of plasma channel behind the front of the ionization wave is faster than the polarization of plasma before the front of FIW:

$$
\tau_{F I W}=R_{f} / V_{F I W}<<\tau_{p o l}
$$


here $R_{f}$ is a characteristic filament radius, $V_{F I W}$ is the velocity of the filament. Otherwise, a rapid broadening of the front of ionization wave will be observed.

The polarization time $\tau_{\text {pol }}$ of a plasma channel of length $\mathrm{L}$ and radius $\mathrm{R}$ is [24]

$$
\tau_{p o l}=\frac{1}{4 \pi \sigma} \cdot\left(\frac{L}{2 R}\right) / \ln \left(\frac{L}{R}\right)
$$

where $\sigma$ is the conductivity of the channel. Under the domination of electron-neutral collisions over electron-ion collisions, $\sigma \sim n_{e} / N[24]$.

At $P=5$ atm and the following parameters of the streamer channel: the length $L=2 \mathrm{~mm}$, the radius $R=100 \mu \mathrm{m}$ and the electron number density $n_{e} \approx 5 \cdot 10^{14} \mathrm{~cm}^{-3}$ [1], we obtain the polarization time of such a channel $\tau_{\text {pol }} \approx 0.3$ ns. Therefore, to prevent the broadening of the front of the filament when the filament propagates as a fast ionization wave (FIW) over the streamer channels, the velocity of the wave should be $V_{F I W}>>3 \cdot 10^{6} \mathrm{~cm} / \mathrm{s}$, in agreement with the experimental data on velocity of the filaments.

\section{Conclusions}

In this work, a detailed study of properties of plasma in the filamentary nanosecond surface dielectric barrier discharge at high pressure in air and nitrogen, $P=5-11$ bar, and high voltages, $U=30-50 \mathrm{kV}$ on the electrode, is performed.

The velocity of filaments changes with the applied voltage but almost does not change with pressure. For pressure and voltage ranges indicated above, the velocity of filaments stays between $0.3 \mathrm{~mm} / \mathrm{ns}$ and $0.5 \mathrm{~mm} / \mathrm{ns}$. The energy per filament per unit length, filament radius and the specific delivered energy were measured. The specific delivered energy in the filamentary plasma measured from experiment can be as high as $\omega=7.4 \mathrm{eV} /$ particle. Synchronized in time electron number density $n_{e}(t)$ and electron

temperature $T_{e}$ were measured by means of Stark broadening and analysis of the relative intensity of $\mathrm{N}^{+}$lines respectively. The electron density in the discharge was found to be equal to $n_{e}=5 \cdot 10^{18} \mathrm{~cm}^{-3}$, decreasing in the near afterglow with a typical decay time tens of nanoseconds. the electron temperature decays from $T_{e}>3 \mathrm{eV}$ in the discharge to $T_{e}=1.7 \mathrm{eV}$ in the near afterglow.

Suggested model assumes that the initiation of the filaments takes place always near the electrode, after the start of the streamers from the electrode. In the case of the cathode a reason of formation of the high electron density near the electrode can be a field emission instability. The region with a high electron density is formed near the cathode, the potential on the front of this region being significantly higher than the potential of the streamer plasma. The sharp gradient of electrical potential near the electrode initiates the wave of a potential gradient, or the fast ionization wave (FIW) propagating from the cathode and additionally charging the surface of the dielectric by the streamer zone formed at the heads of the filaments.

High electric fields during a short period of time, less than $1 \mathrm{~ns}$, in the front of the filament, are responsible for efficient ionization. Stepwise ionization and dissociation 
from electronically excited states of molecular nitrogen leads to fast increase of the electron density, dissociation degree and gas heating. Approaching to the state close to the LTE explains the anomalously slow plasma decay in the afterglow, tens of nanoseconds: the electron density corresponds to the value of gas temperature at the given time instant, so the dynamics of plasma decay is determined mainly by gas cooling.

\section{Acknowledgements}

The work was partially supported by LabEx Plas@Par, French National Research Agency (ASPEN Project) and the French-Russian international laboratory LIA KaPPA

"Kinetics and physics of pulsed plasmas and their afterglow" (RFBR project 17-5216001 and CNRS financial and organization support). The support of China Scholarship Council (CSC) for Chenyang Ding is gratefully acknowledged. The authors are thankful to Ali Mahjoub and Bruno Dufour for engineering support.

\section{References}

[1] Starikovskiy A and Aleksandrov N 2013 Plasma-assisted ignition and combustion Progress in Energy and Combustion Science 39 61-110

[2] Starikovskaia S 2014 Plasma-assisted ignition and combustion: nanosecond discharges and development of kinetic mechanisms Journal of Physics D: Applied Physics 47353001

[3] Ju Y and Sun W 2015 Plasma assisted combustion: Dynamics and chemistry Progress in Energy and Combustion Science 48 21-83

[4] Starikovskiy A, Aleksandrov N and Rakitin A 2012 Plasma-assisted ignition and deflagration-todetonation transition Phil. Trans. R. Soc. A 370 740-773

[5] Zhu Y, Anand V, Jodele J, Knight E, Gutmark E J and Burnette D 2017 Plasma-assisted rotating detonation combustor operation 53rd AIAA/SAE/ASEE Joint Propulsion Conference p 4742

[6] Gray J A and Lacoste D A 2019 Enhancement of the transition to detonation of a turbulent hydrogen-air flame by nanosecond repetitively pulsed plasma discharges Combustion and Flame $199258-266$

[7] Leonov S B, Adamovich I V and Soloviev V R 2016 Dynamics of near-surface electric discharges and mechanisms of their interaction with the airflow Plasma Sources Science and Technology 25063001

[8] Pai D Z, Lacoste D A and Laux C O 2010 Nanosecond repetitively pulsed discharges in air at atmospheric pressure - the spark regime Plasma Sources Science and Technology 19065015

[9] Janda M, Machala Z, Dvonč L, Lacoste D and Laux C O 2015 Self-pulsing discharges in pre-heated air at atmospheric pressure Journal of Physics D: Applied Physics 48035201

[10] Van der Horst R, Verreycken T, Van Veldhuizen E and Bruggeman P 2012 Time-resolved optical emission spectroscopy of nanosecond pulsed discharges in atmospheric-pressure $\mathrm{N}_{2}$ and $\mathrm{N}_{2} / \mathrm{H}_{2} \mathrm{O}$ mixtures Journal of Physics D: Applied Physics 45345201

[11] Janda M, Hoder T, Sarani A, Brandenburg R and Machala Z 2017 Cross-correlation spectroscopy study of the transient spark discharge in atmospheric pressure air Plasma Sources Science and Technology 26055010

[12] Orrière T, Moreau E and Pai D Z 2018 Ionization and recombination in nanosecond repetitively pulsed microplasmas in air at atmospheric pressure Journal of Physics D: Applied Physics $\mathbf{5 1}$ 494002

[13] Minesi N, Stepanyan S A, Mariotto P B, Stancu G D and Laux C O 2019 On the arc transition mechanism in nanosecond air discharges AIAA Scitech 2019 Forum p 0463 
[14] Lo A, Cessou A, Lacour C, Lecordier B, Boubert P, Xu D, Laux C and Vervisch P 2017 Streamerto-spark transition initiated by a nanosecond overvoltage pulsed discharge in air Plasma Sources Science and Technology 26045012

[15] Stepanyan S, Starikovskiy A Y, Popov N and Starikovskaia S 2014 A nanosecond surface dielectric barrier discharge in air at high pressures and different polarities of applied pulses: transition to filamentary mode Plasma Sources Science and Technology 23045003

[16] Shcherbanev S, Khomenko A Y, Stepanyan S, Popov N and Starikovskaia S 2016 Optical emission spectrum of filamentary nanosecond surface dielectric barrier discharge Plasma Sources Science and Technology 26 02LT01

[17] Ding C, Khomenko A Y, Shcherbanev S A and Starikovskaia S M 2019 Filamentary nanosecond surface dielectric barrier discharge. experimental comparison of streamer-to-filament transition for positive and negative polarities Plasma Sources Science and Technology Submitted

[18] Shcherbanev S A, Popov N A and Starikovskaia S M 2017 Ignition of high pressure lean h2: air mixtures along the multiple channels of nanosecond surface discharge Combustion and Flame $176272-284$

[19] Anikin N, Starikovskaia S and Starikovskii A Y 2002 Polarity effect of applied pulse voltage on the development of uniform nanosecond gas breakdown Journal of Physics D: Applied Physics 352785

[20] Soloviev V, Krivtsov V, Shcherbanev S and Starikovskaia S 2016 Evolution of nanosecond surface dielectric barrier discharge for negative polarity of a voltage pulse Plasma Sources Science and Technology 26014001

[21] Soloviev V R and Krivtsov V M 2018 Numerical modelling of nanosecond surface dielectric barrier discharge evolution in atmospheric air Plasma Sources Science and Technology 27114001

[22] Kramida A, Yu Ralchenko, Reader J and and NIST ASD Team 2018 NIST Atomic Spectra Database (ver. 5.6.1), [Online]. Available: https://physics.nist.gov/asd [2018, March 25]. National Institute of Standards and Technology, Gaithersburg, MD.

[23] Mintoussov E, Pendleton S, Gerbault F, Popov N and Starikovskaia S 2011 Fast gas heating in nitrogen-oxygen discharge plasma: II. energy exchange in the afterglow of a volume nanosecond discharge at moderate pressures Journal of Physics D: Applied Physics $4 \mathbf{4} 285202$

[24] Raizer Y P 1991 Gas discharge physics (Verlag Berlin Heidelberg: Springer) ISBN 978-3-64264760-4

[25] Andreev S and Gavrilova T 1974 Investigation of a pulsed stabilized discharge in air at a pressure above 100 atm High temperature 12 1138-1142

[26] Park C 1993 Review of chemical-kinetic problems of future nasa missions. I-Earth entries Journal of Thermophysics and Heat transfer 7 385-398

[27] Hagelaar G and Pitchford L 2005 Solving the boltzmann equation to obtain electron transport coefficients and rate coefficients for fluid models Plasma Sources Science and Technology 14722

[28] Phelps A and Pitchford L 1985 Anisotropic scattering of electrons by $\mathrm{N}_{2}$ and its effect on electron transport Physical Review A 312932

[29] 2013 SIGLO database http://www.lxcat.laplace.univ-tlse.fr [2013, June 4].

[30] Alves L 2014 The ist-lisbon database on lxcat Journal of Physics: Conference Series vol 565 (IOP Publishing) p 012007

[31] Bacri J and Medani A 1982 Electron diatomic molecule weighted total cross section calculation: III. main inelastic processes for $\mathrm{N}_{2}$ and $\mathrm{N}_{2}{ }^{+}$Physica $B+C 112$ 101-118

[32] Capitelli M, Colonna G and D'Angola A 2011 Fundamental aspects of plasma chemical physics: thermodynamics vol 66 (Springer Science \& Business Media)

[33] Parkevich E, Ivanenkov G, Medvedev M, Khirianova A, Selyukov A, Agafonov A, Mingaleev A, Shelkovenko T and Pikuz S 2018 Mechanisms responsible for the initiation of a fast breakdown in an atmospheric discharge Plasma Sources Science and Technology 27 11LT01

[34] Khirianova A, Parkevich E and Tkachenko S 2018 Features of interferometry of small-sized plasma channels obtained during nanosecond discharge Physics of Plasmas 25073503 
[35] Korolev Y D and Mesyats G A 1998 Physics of Pulsed Breakdown in Gases (Ekaterinburg: Ural Division of the Russian Academy of Science) ISBN 5769107790

[36] Levko D and Raja L L 2018 High-voltage microdischarge as a source of extreme density plasma Physics of Plasmas 25013509

[37] Orrière T 2018 Confinement microméetrique de déecharges pulsées nanosecondes dans l'ais a pression atmosphérique et effets électro-aérodynamiques Ph.D. thesis University of Poitiers France (in French)

[38] Vasilyak L, Kostyuchenko S, Kudryavtsev N and Filyugin I 1994 High-speed ionization waves at an electric breakdown Phys.-Uspekhi 163 263-286 